\title{
Review
}

\section{The oncolytic virotherapy treatment platform for cancer: Unique biological and biosafety points to consider}

\author{
Richard Vile, ${ }^{1}$ Dale Ando, ${ }^{2}$ and David Kirn ${ }^{3,4}$ \\ ${ }^{1}$ Molecular Medicine Program, Mayo Clinic, Rochester, Minnesota, USA; ${ }^{2}$ Cell Genesys Corp., Foster City, \\ California, USA; ${ }^{3}$ Department of Pharmacology, Oxford University Medical School, Oxford, UK; and ${ }^{4}$ Kirn \\ Oncology Consulting, San Francisco, California, USA.
}

\begin{abstract}
The field of replication-selective oncolytic viruses ( virotherapy) has exploded over the last 10 years. As with many novel therapeutic approaches, initial overexuberance has been tempered by clinical trial results with first-generation agents. Although a number of significant hurdles to this approach have now been identified, novel solutions have been proposed and improvements are being made at a furious rate. This article seeks to initiate a discussion of these hurdles, approaches to overcome them, and unique safety and regulatory issues to consider.
\end{abstract}

Cancer Gene Therapy (2002) 9, 1062 - 1067 doi:10.1038/sj.cgt.7700548

Keywords: oncolytic; virotherapy; experimental therapeutics; cancer

$\mathrm{N}$ ew cancer treatments are needed. These agents must have novel mechanisms of action and thereby lack cross - resistance with currently available treatments. Viruses have evolved to infect, replicate in, and kill human cells through diverse mechanisms. Clinicians treated hundreds of cancer patients with a spectrum of wild-type viruses over the last century, but the approach was temporarily abandoned due to toxicity and due to initial enthusiasm over the advent of chemotherapy. ${ }^{1}$ With the discovery of recombinant DNA technology and the genetic mechanisms leading to cancer, genetic engineering of viruses to safely target cancers became possible. The initial approach was to make the therapeutic gene-expressing viral vectors replicationincompetent (i.e., gene therapy). Although still promising, this approach has not led to an approved anticancer agent to date. The major limitation with replication-incompetent gene therapy for cancer has been the inefficiency of gene delivery to cancer cells in vivo.

Following the first description of a virus engineered to replicate selectively in dividing cells almost a decade ago, ${ }^{2}$ the field of viral therapy for cancer (virotherapy) has been reborn and has expanded dramatically. This approach seeks to build on the positive features of gene therapy with replication-incompetent viruses (e.g., unique and selective cancer killing mechanisms) while overcoming the major

Received September 24, 2002

Address correspondence and reprint requests to: Dr David Kirn. E-mail: davidhkirn@sbcglobal.net limitation of levels of delivery to cancer cells in a solid tumor mass. Over 10 different virotherapy agents have entered, or will soon be entering, clinical trials; one such adenovirus (dl1520) has entered a Phase III clinical trial in recurrent head and neck carcinoma. Over 10 different biotechnology and large pharmaceutical companies now have active virotherapy programs. There is, therefore, a tremendous amount of activity in this field. However exciting the therapeutic potential is, this approach also presents unique scientific, regulatory, and biosafety issues. This article seeks to initiate a public dialogue on (a) approaches to overcome the potential hurdles to effective virotherapy, and (b) the safety and regulatory issues to consider that are unique to virotherapy.

\section{Desirable features for oncolytic virotherapy agents}

When considering a virus species for development as an oncolytic therapy, a number of efficacy, safety, and manufacturing issues need to be assessed. The virus should infect, replicate in, and destroy human tumor cells, ideally including noncycling cancer cells. The parental virus should preferably cause only mild, well - characterized human disease( s ). Alternatively, deletion mutants that are themselves nonvirulent should be considered. Nonintegrating viruses have potential safety advantages in that unpredicted events caused by genomic integration are avoided. A genetically stable virus is desirable from both safety and manufacturing standpoints. Genetic approaches to prevent viral replication in essential normal tissues are critical and a secondary mechanism to 
inactivate the virus should ideally be available (i.e. an "offswitch"). Finally, the virus must be amenable to high-titer production and purification under Good Manufacturing Practices (GMP) guidelines for clinical studies.

\section{Mechanisms of tumor selectivity}

Viruses have evolved to dramatically alter the phenotype of the infected cell to maximize their own replication and survival. The cellular changes induced by viral infection are often strikingly similar to the cellular changes acquired during carcinogenesis (e.g., p53 tumor suppressor protein inactivation, inhibition of apoptosis). Given this genetic convergence, it is not surprising that many viruses inherently grow preferentially in tumor cells and/or that viruses can be engineered for tumor selectivity. Five general mechanistic approaches to tumor-selective replication have been described to date. These include (a) the use of viruses with inherent tumor selectivity (e.g., NDV, reovirus, VSV, autonomous parvovirus $) ;^{3-6}$ (b) deletion of entire genes (e.g., HSV, adenovirus, vaccinia) ${ }^{7-10}$ or (c) functional gene regions (e.g., adenovirus, poliovirus $)^{11-13}$ that are necessary for efficient replication and/or toxicity in normal cells but are expendable in tumor cells; (d) engineering of tumor/ tissue-specific promoters into viruses to limit expression of gene(s) necessary for replication to cancer cells (e.g., adenovirus, HSV ); ${ }^{14}$ and (e ) modification of the viral coat to target uptake selectively to tumor cells (e.g., adenovirus, poliovirus). ${ }^{15,16}$ Each of these approaches has potential advantages and disadvantages.

\section{Potential hurdles to be overcome}

Potential limitations to virotherapy have been identified. As the majority of research to date have been with adenoviruses, more is known about the limitations with this virus species than for others. First, although viruses rapidly spread in cell culture monolayers, viral spread within a solid tumor mass can be limiting. ${ }^{17,18}$ In fact, mathematical modeling of the "race" between viral oncolysis and spread versus tumor cell proliferation and outgrowth demonstrates that the infection of the tumor must be diffuse throughout the tumor in order to control it; injection of the tumor "core" or periphery only results in tumor "escape". 19 The relative inefficiency of viral spread may relate to their relatively large sizes (e.g., $90 \mathrm{~nm}$ for adenovirus), dwarfing antitumoral chemicals, peptides, and even antibodies. Potential physical limitations to viral spread include fibrosis, intermixed normal cells (up to half of the cells within some tumors), basement membranes, cellto-cell barriers, and necrotic regions. Viral mutants that are more efficiently released and spread in tumors have been identified; these include $E 1 A-C R 2$ mutant $^{11}$ and $E 1 B-19$ $k D a$ mutant adenoviruses, ${ }^{20,21}$ and mutants that overexpress the $E 3-11.6$ gene product. $^{22}$ In addition, certain virus species spread more efficiently than others. Adenovirus, for example, spreads slowly because it remains primarily intracellular and appears to spread through apoptotic bodies; its replication cycle typically lasts for $48-72$ hours. $^{23}$ In contrast, some viruses are actively pumped out of the in- fected cell (e.g., vaccinia) and others kill the infected cell faster (e.g., HSV, vaccinia). ${ }^{24}$ In particular, viruses with extracellular forms (e.g., vaccinia) may have distinct advantages in intratumoral spread.

Insufficient expression of viral receptors on target tumors has also been shown to limit efficacy. ${ }^{25}$ Expression of the CAR receptor for adenovirus, for example, appears to vary dramatically not only between tumor and tissue types, but also between different stages in a given tumor type. ${ }^{26}$ Phase I/II clinical trials of the Onyx-015 adenovirus, for example, demonstrated reproducible infection of head and neck cancers but not pancreatic or ovarian carcinomas. ${ }^{27-29}$ Effective retargeting of viral agents will probably require both ablation of their normal tissue tropism/receptorbinding and the subsequent introduction of new receptorbinding motifs. Although most of these works have been done with adenovirus, ${ }^{15,25,30,31}$ enveloped viruses such as measles can also be modified in this fashion ( see review by $\mathrm{S}$ Russell, this volume).

The immune response will presumably limit ongoing viral replication and spread in immunocompetent patients eventually, ${ }^{32}$ although immune responses may also lead to enhanced antitumoral effects. ${ }^{33}$ The route of viral administration will be a critical determining factor. Neutralizing antibodies do not appear to block efficacy following intratumoral injection in mice or patients with a wide variety of different viruses. ${ }^{10,18,28,34,35}$ Viruses that spread through efficient cell-cell fusion may avoid contact with antibodies completely (e.g., measles). In addition, targeted viral delivery through the artery perfusing a tumor mass (e.g., hepatic artery perfusion of colorectal liver metastases) appears to be feasible despite high-level antibody titers. ${ }^{36,37}$ The role of neutralizing antibodies following intravenous administration remains to be determined. It is important to remember that humans' immune systems did not evolve to clear intravenous boluses of virus at doses up to $\geq 10^{12}$ particles as have been used in clinical trials. ${ }^{38}$ Despite the development of neutralizing antibodies following treatment, intravenous virus has been delivered to solid tumors in patients on Phase I clinical trials with both adenovirus and NDV. ${ }^{38,39}$ Methods to reduce neutralizing antibody formation and/or titers have been proposed. These have included ablating B-lymphocyte function (e.g., using anti-CD20 antibody therapy with rituxan) or using plasma pheresis columns to elute off antibodies to adenovirus. These approaches have not been tested in the clinic to date. The lack of an immunocompetent model for replicationcompetent adenoviruses has been a critical limitation for this approach. ${ }^{40}$ Vaccinia virus has forms that are inherently resistant to neutralizing antibodies and/or complementmediated clearance. ${ }^{41}$

Although intravenous adenovirus, HSV, reovirus, and NDV can have antitumoral efficacy in immunodeficient mice, ${ }^{42}$ the inefficiency of delivery to distant metastatic sites even in the absence of an immune response is still a major hurdle. Virus delivery to solid tumors following intravenous administration was feasible in clinical trials as described above, ${ }^{38,39}$ but reproducible antitumoral efficacy data must await Phase II trials. Rapid clearance of viruses from the bloodstream can result from reticuloendothelial cell uptake, 
antibody binding, or complement-mediated effects, depending on the virus species. ${ }^{42,43}$ Some poxvirus vectors such as vaccinia appear to target systemic metastases following intravenous administration; the mechanism of this systemic tumor targeting is unclear to date. ${ }^{44,45}$ One mechanism may be through the incorporation of complement resistance proteins into their outer membranes. In addition to using complement-resistant viruses, other groups have proposed the pretreatment with cyclophosphamide to knock out preexisting "natural" antibodies to the viral agent. ${ }^{43}$ Measles virus was initially developed as a virotherapy agent for lymphoid malignancies due to its inherent systemic targeting of lymphoid cells ( $\mathrm{S}$ Russell, this volume); additional research has demonstrated the potential of a measles vaccine strain (MV-SPUD) to selectively destroy epithelial cell-derived carcinomas as well ( $\mathrm{S}$ Russell, this volume).

Toxicity must be a primary concern with any experimental therapeutic agent, and virotherapy agents are no different. The toxicity of greatest concern will be dependent on the virus strain used, the specific genetic changes made, and the route/dose of administration. Adenovirus, for example, appears to be capable of inducing reversible liver inflammation and low-grade disseminated intravascular coagulation (DIC) at doses of $\geq 10^{12}$ particles in cancer patients. ${ }^{37,46}$ Despite the death of a noncancer patient at the University of Pennsylvania due to DIC and liver failure following hepatic artery infusion of a replication-incompetent adenovirus, cancer patients treated with intravascular adenovirus have tolerated the agents well; no treatment-related deaths have been reported ${ }^{46}$ (Reid and Kirn, this volume). Oncolytic HSV agents such as G207 were engineered to decrease their neurotoxicity and the risk of encephalitis ${ }^{47,48}$ (S Rabkin, this volume).

Viruses can be "armed" to express exogenous therapeutic genes including cytokines or prodrug-activating enzymes $^{10,49-55}$ ( $\mathrm{T}$ Hermiston, this volume). Although these combination genetic therapeutic agents hold great promise, in some cases, the biology of the virus lifecycle can be adversely affected (e.g., prodrug-activating enzyme therapy). ${ }^{56}$ In addition, some viruses have a very limited transgene-carrying capacity in the context of a replicationcompetent virus with most genes intact; adenovirus, for example, can only efficiently package a genome that is approximately $5 \%$ over its normal size $(1.5-2 \mathrm{~kb}$ total $)$. Other viral species can accept anywhere from $5+\mathrm{kb}$ (e.g., measles, NDV) to over $15 \mathrm{~kb}$ (e.g., HSV, vaccinia) of foreign DNA; thus, with these viral species, the regulated expression of multiple therapeutic and safety genes becomes a possibility. At this time, reovirus engineering has not been reported, but groups are working to resolve this hurdle ( $\mathrm{S}$ Russell, this volume).

\section{Risk management: patients, patient contacts, and the general public}

Virotherapy agents raise new biosafety and risk management issues. The risk assessment for trials with these agents must not only take into account potential risks to the treated patient but to patient contacts and the general public. For cancer patients with refractory, end-stage disease, the riskbenefit ratio has supported the development of extremely toxic treatment approaches, some of which routinely result in severe morbidity and mortality (e.g., allogeneic bone marrow transplant); this is acceptable because long-term remissions are possible. In the cancer treatment setting, treatment-related toxicity is frequently expected. Virotherapy agents may be substantially less toxic than standard chemotherapeutics (this must be proven on a case-by-case basis ). Nevertheless, there will always be a risk of toxicity during clinical trial testing, and this may be acceptable in patients with terminal cancer. Once an acceptable safety profile has been demonstrated in end-stage, refractory patients, it may be ethical to move into earlier-stage patient populations. ${ }^{32}$ The treatment of earlier-stage patients (i.e., curable or good-prognosis patients) may require previous use of the agent in end-stage patients and/or localized administration initially (e.g., intratumoral, intravesicle). Finally, use in combination with standard chemotherapy and/or radiotherapy will generally require prior experience with the virus as a single agent. ${ }^{32}$

The first step in the preclinical Good Laboratory Practice (GLP) toxicity assessment is the selection of relevant animal models to test replication in vivo, toxicity, and biodistribution. Often times, these parameters can be tested in animal models, but due to species-specific tropism differences, relevant models are not always available. The additive usefulness of safety studies in primates should be considered. Viral shedding and mobilization/recombination with wildtype virus are other issues that may be addressed in these studies.

Important factors for safety consideration prior to trials with new agents include the spectrum of disease caused by the parental viral strain, the level of preexisting immunity to the parental virus in the population, the ability of the virus to evade the immune response, and the tropism of the virus. If tropism has been modified, has the spectrum of cells that are infectable been narrowed (to avoid infection of normal tissues) or are previously resistant tissue types now infectable (raising the risk of a new spectrum of disease)? What is the risk of reversion to the wild-type strain? The risk of reversion to wild-type virus can be decreased by engineering multiple selectivity mechanisms and safety features into the agent (e.g., HSV G207). Are effective antiviral agents available? Viruses expressing therapeutic transgenes raise additional questions. Has the viral vector itself been demonstrated to be safe and selective in patients in the absence of the transgene? What is the likely toxicity of transgene expression in normal tissues? For example, a prodrugactivating enzyme may have little or no toxicity in the absence of the relevant prodrug, whereas an inflammatory cytokine such as tumor necrosis factor might lead to serious local or even systemic toxicities. If reversion to a wild-type, nonselective virus were to occur, would the transgene still be expressed? What would be the consequences of a recombination of the engineered virus with a related wild-type virus in the population?

It will be important to obtain data on viral replication, biodistribution, and shedding to the environment in early phase clinical trials. Long-term follow-up programs to 
assess latency may be appropriate in many cases. Classical infectious disease vertical and horizontal transmission studies may subsequently be done depending on vector class; collaborations between different sponsors in the field would be useful to maximize the available database for the assessment of environmental impact. RAC or NIH sponsorship of such studies would be useful in cases of academic institution-sponsored trials.

The Oslerian adage that the physician must first "do no harm" carries substantial additional weight when one considers the possibility of an adverse effect on the population at large. Investigators in our field must take all precautions that are reasonably necessary to avoid the release of potentially harmful agents into the environment. The following approaches should be considered when any new virotherapy agent is engineered. First, are effective antiviral agents available for the virus? Should drug sensitivity enzyme genes (e.g., prodrug-activating enzymes such as thymidine kinase) be inserted in order to increase virus sensitivity to available agents? The need for an antiviral strategy will increase as the potential virulence of the virus increases (e.g., virulent clinical isolates, viruses expressing toxic transgenes) and as the preexisting immunity to the virus in the general population decreases. Second, is it advisable to engineer potentially toxic transgenes into oncolytic viruses prior to proof of safety and tumor selectivity of the parental virus in clinical trials? Likewise, is it advisable to alter the tropism of oncolytic viruses to include new, previously unaffected tissues prior to proof of selectivity and safety in patients with a nonretargeted version of the virus first? Once again, if the tropism of the virus has simply been restricted to a subset of the virus' normal tissue range, then safety may be increased.

In addition to genetic engineering, the safety of these agents should be improved by reducing exposure of the public. Exposure of patient contacts can be reduced through patient isolation (e.g., negative airflow may be considered). The first patients treated may be isolated for a predetermined number of days or until viral shedding in bodily fluids is no longer detectable. Once the safety of high doses has been demonstrated in patients on clinical trials, particularly if the virus was demonstrated to spread systemically through the bloodstream, the safety concerns related to incidental contact with relatively small amounts of the virus will be much less.

\section{Time for head-to-head comparisons?}

With a great diversity of viral families now described with some degree of tumor selectivity, at some point in time an important question must surely raise its controversial head above the parapet. If so many viruses are potentially useful for targeting cancer cells, whether genetically altered or not, which one is the best? This is not simply a question designed to satisfy intellectual curiosity. It has obvious relevance to patient care, clearly influences the number of clinical trials that will be carried out, and, perharps, will avoid the instigation of experimental therapies that turn out to have no benefit or, even more importantly, have some negative toxicities. However, although the literature continues to amass descriptions of new and improved versions of different replicating viral systems, the lack of any meaningful head-to-head comparisons between these systems is distinctly notable by its absence. Of course, there are several very real reasons why this should be the case. Groups working on one viral type may have insufficient expertise to use other systems for such comparative studies; different vectors are used in different disease types and settings (local injection $v s$ systemic administration). Therefore, it becomes genuinely difficult to compare the safety and efficacy of different viruses. However, a somewhat more cynical view can also be aired. Thus, it may be that, predominantly for commercial reasons, comparisons of potential products are not in the best interests of shareholders. As soon as any comparison is made with other market competitiors, there may be a loser, in which case companies and jobs can be lost. However, despite the technical arguments, there are undoubtedly situations where different replicating vectors can be genuinely compared and contrasted for efficacy. Similar comparisons have been carried out in other fields where cancer therapeutic candidates such as cytokines have been put to the test side by side, ${ }^{57}$ although it is far from clear that such studies would be done today. Nonetheless, should the field not be thinking at this stage of rationalizing its approach to the testing of different replicating viruses for the virotherapy of cancer? Perhaps one way forward would be through the formation of large collaborative groups in which there is clear incentive for different viral systems to be tested in models for which there is widespread agreement on design and for which there is multiauthor credit. Such groups already exist for the testing of new therapies in other areas of oncology, designed to benefit the patient and get the most effective therapies to the most patients in the shortest period of time. Perhaps it is time for a similar collaborative effort in the field of virotherapy for cancer.

\section{Summary}

Virotherapy holds great promise as a novel treatment platform for cancer. Advantages include the potential lack of cross-resistance with standard therapies and their ability to cause tumor destruction by numerous mechanisms. However, significant hurdles have been identified for virotherapy agents, in particular for adenovirus to date. Novel approaches are being explored to overcome these limitations with adenovirus. Other viral species have potential advantages as oncolytic agents that may be exploited in the future (e.g., vaccinia virus, measles virus, and others). In addition, unique safety issues have been raised by all oncolytic viruses. These issues must be addressed in a timely fashion because the study of replicating agents for tumor therapy is rapidly evolving and extending into clinical trials. New risk management guidelines are needed for this exciting cancer treatment platform.

\section{References}

1. Southam CM. Present status of oncolytic virus studies. Ann NY Acad Sci. 1960:656-673. 
2. Martuza RL, Malick A, Markert JM, Ruffner KL, Coen DM. Experimental therapy of human glioma by means of a genetically engineered virus mutant. Science. 1991;252:854856.

3. Lorence RM, Rood PA, Kelley KW. Newcastle disease virus as an antineoplastic agent: induction of tumor necrosis factoralpha and augmentation of its cytotoxicity. J Natl Cancer Inst. 1988;80:1305-1312.

4. Coffey M, Strong J, Forsyth P, Lee P. Reovirus therapy of tumors with activated ras pathway. Science. 1998;282:13321334.

5. Stojdl DF, et al. Exploiting tumor-specific defects in the interferon pathway with a previously unknown oncolytic virus. Nat Med. 2000;6:821-825.

6. Rommelaere J, Cornelis JJ. Antineoplastic activity of parvoviruses. J Virol Methods. 1991;33:233-251.

7. Martuza RL, Malick A, Markert JM, Ruffner KL, Coen DM. Experimental therapy of human glioma by means of a genetically engineered virus mutant. Science. 1991;252:854-856.

8. Bischoff JR, et al. An adenovirus mutant that replicates selectively in p53-deficient human tumor cells [see comments]. Science. 1996;274:373-376.

9. Heise C, et al. ONYX-015, an E1B gene-attenuated adenovirus, causes tumor-specific cytolysis and antitumoral efficacy that can be augmented by standard chemotherapeutic agents [see comments]. Nat Med. 1997;3:639-645.

10. Mastrangelo M, Eisenlohr L, Gomella L, Lattime E. Poxvirus vectors: orphaned and underappreciated. J Clin Invest. 2000; 105:1031-1034.

11. Heise C, et al. An adenovirus E1A mutant that demonstrates potent and selective antitumoral efficacy. Nat Med. 2000;6: $1134-1139$.

12. Fueyo J, et al. A mutant oncolytic adenovirus targeting the $\mathrm{Rb}$ pathway produces anti-glioma effect in vivo. Oncogene. 2000; 19:2-12.

13. Gromeier M, Lachmann S, Rosenfeld MR, Gutin PH, Wimmer E. Intergeneric poliovirus recombinants for the treatment of malignant glioma [see comments]. Proc Natl Acad Sci USA. 2000;97:6803-6808.

14. Miyatake S, Iyer A, Martuza RL, Rabkin SD. Transcriptional targeting of herpes simplex virus for cell-specific replication. J Virol. 1997;71:5124-5132.

15. Wickham TJ, Shears TEL, Roelvink $P$, et al. Increased in vitro and in vivo gene transfer by adenovirus vectors containing chimeric fiber proteins. J Virol. 1997;71:8221-8229.

16. Alemany R, Curiel DT. The development of replicative adenoviruses for cancer therapy. Clin Cancer Res. 2000;6:33953399.

17. Heise C, Williams A, Olesch J, Kirn D. Efficacy of a replication-competent adenovirus (ONYX-015) following intratumoral injection: intratumoral spread and distribution effects. Cancer Gene Ther. 1999;6:499-504.

18. Ganly I, et al. A phase I study of Onyx - 015, an E1B attenuated adenovirus, administered intratumorally to patients with recurrent head and neck cancer. Clin Cancer Res. 2000;6:798806.

19. Wu JT, Byrne HM, Kirn DH, Wein LM. Modeling and analysis of a virus that replicates selectively in tumor cells. Bull Math Biol. 2001;63:731-768.

20. Chinnadurai G. Adenovirus $2 \mathrm{Ip}^{+}$locus codes for a $19 \mathrm{kD}$ tumor antigen that plays an essential role in cell transformation. Cell. 1983;33:759-766.

21. Sauthoff H, Heitner S, Rom W, Hay J. Deletion of the adenoviral E1B-19 kD gene enhances tumor cell killing of a replicating adenoviral vector. Hum Gene Ther. 2000;11:379-388.

22. Doronin K, et al. Tumor-specific, replication-competent ade- novirus vectors overexpressing the adenovirus death protein. J Virol. 2000;74:6147-6155.

23. Shenk T. In: Fields K, Howley P, eds. Fields Virology. Philadelphia: Lippincott-Raven; 1996:2135-2137.

24. Smith GL, Vanderplasschen A. Extracellular enveloped vaccinia virus. Entry, egress, and evasion. Adv Exp Med Biol. 1998;440:395-414.

25. Douglas J, Kim M, Sumerel L, Carey D, Curiel D. Efficient oncolysis by a replicating adenovirus in vivo is critically dependent on tumor expression of primary Ad receptors. Cancer Res. 2001;61:813-817.

26. Rauen KA, et al. Expression of the coxsackie adenovirus receptor in normal prostate and in primary and metastatic prostate carcinoma: potential relevance to gene therapy. Cancer Res. 2002;62:3812-3818.

27. Vasey P, Shulman L, Gore M, Kirn D, Kaye S. Phase I trial of intraperitoneal Onyx -015 adenovirus in patients with recurrent ovarian carcinoma. Proc Am Soc Clin Oncol. 2000;19:1512. (Abstract).

28. Nemunaitis J, et al. Selective replication and oncolysis in $\mathrm{p} 53$ mutant tumors with Onyx-015, an E1B-55 kD gene-deleted adenovirus, in patients with advanced head and neck cancer: a phase II trial. Cancer Res. 2000;60:6359-6366.

29. Mulvihill S, Warren R, Kirn D. Safety and feasibility of injection with an E1B-55 kD gene-deleted replicationselective adenovirus Onyx-015 into primary carcinomas of the pancreas: a phase I trial. Gene Ther. 2001;8:308-315.

30. Roelvink P, Mi G, Einfeld D, Kovesdi I, Wickham T. Identification of a conserved receptor-binding site on the fiber proteins of CAR-recognizing adenoviridae. Science. 1999;286: $1568-1571$.

31. Suzuki K, et al. A replicative adenovirus with enhanced infectivity shows improved oncolytic potency. Clin Cancer Res. 2001;7:120-126.

32. Kirn D. Clinical research results with $d l 1520$ (Onyx-015), a replication-selective adenovirus for the treatment of cancer: what have we learned? Gene Ther. 2001;8:89-98.

33. Toda $T$, et al. Systemic antitumor immunity in experimental brain tumor therapy using a multimutated, replication-competent herpes simplex virus. Hum Gene Ther. 1999;10:27412755.

34. Herrlinger U, et al. Pre-existing herpes simplex virus 1 (HSV1 ) immunity decreases, but does not abolish, gene transfer to experimental brain tumors by a HSV-1 vector. Gene Ther. 1998; 5:809-819.

35. Merritt J, et al. Clinical phase I experience with INGN 201 (Ad-p53) in non-small cell lung cancer and head and neck cancer. Cancer Gene Ther. 1997;4:S12. (Abstract).

36. Reid $\mathrm{T}$, et al. Hepatic artery infusion of Onyx-015, a replication-selective adenovirus, in combination with $5-\mathrm{FU} /$ leucovorin for gastrointestinal carcinoma metastatic to the liver: a phase I/II clinical trial. Proc Am Soc Clin Oncol. 2000; 19:953. (Abstract).

37. Reid $\mathrm{T}$, et al. Intra-arterial administration of a replicationselective adenovirus (d11520) in patients with colorectal carcinoma metastatic to the liver: a phase I trial. Gene Ther. 2001; $8: 1618-1626$.

38. Nemunaitis $\mathrm{J}$, et al. Intravenous infusion of a replicationselective adenovirus (ONYX-015) in cancer patients: safety, feasibility and biological activity. Gene Ther. 2001;8:746-759.

39. Pecora AL, et al. Phase I trial of intravenous administration of PV701, an oncolytic virus, in patients with advanced solid cancers. J Clin Oncol. 2002;20:2251-2266.

40. Kirn D. Replication-selective oncolytic adenoviruses: virotherapy aimed at genetic targets in cancer. Oncogene. 2000;1: 6660-6668. 
41. Vanderplasschen A, Mathew E, Hollinshead M, Sim RB, Smith GL. Extracellular enveloped vaccinia virus is resistant to complement because of incorporation of host complement control proteins into its envelope. Proc Natl Acad Sci USA. 1998;95:7544-7549.

42. Heise C, Williams A, Xue S, Propst M, Kirn D. Intravenous administration of ONYX-015, a selectively-replicating adenovirus, induces antitumoral efficacy. Cancer Res. 1999;59: $2623-2628$.

43. Ikeda K, Chiocca N. Oncolytic virus therapy of multiple tumors in the brain requires suppression of innate and elicited anti-viral responses. Nat Med. 1999;5:881-887.

44. Gnant MF, Puhlmann M, Alexander HR Jr, Bartlett DL. Systemic administration of a recombinant vaccinia virus expressing the cytosine deaminase gene and subsequent treatment with 5 -fluorocytosine leads to tumor-specific gene expression and prolongation of survival in mice. Cancer Res. 1999;59:3396-3403.

45. Gnant MF, et al. Tumor-specific gene delivery using recombinant vaccinia virus in a rabbit model of liver metastases. J Natl Cancer Inst. 1999;91:1744-1750.

46. Kirn D, Warren R. Liver-directed therapy with p53-targeted adenoviruses: clinical trial results. Surg Clin North Am. 2002. (In press).

47. Mineta T, Rabkin SD, Yazaki T, Hunter WD, Martuza RL. Attenuated multi-mutated herpes simplex virus-1 for the treatment of malignant gliomas. Nat Med. 1995;1:938-943.

48. Markert J, et al. Conditionally replicating herpes simplex virus mutant, G207 for the treatment of malignant glioma: results of a phase I trial. Gene Ther. 2000;7:867-874.

49. Hermiston T. Gene delivery from replication-selective viruses: arming guided missiles in the war against cancer. $J$ Clin Invest. 2000;105:1169-1172.
50. Toda M, Martuza RL, Kojima H, Rabkin SD. In situ cancer vaccination: an IL-12 defective vector/replicationcompetent herpes simplex virus combination induces local and systemic antitumor activity. J Immunol. 1998;160:44574464.

51. Wildner O, Blaese RM, Morris JM. Therapy of colon cancer with oncolytic adenovirus is enhanced by the addition of herpes simplex virus-thymidine kinase. Cancer Res. 1999;59:410413.

52. Freytag SO, Rogulski KR, Paielli DL, Gilbert JD, Kim JH. A novel three-pronged approach to kill cancer cells selectively: concomitant viral, double suicide gene, and radiotherapy [see comments ]. Hum Gene Ther. 1998;9:1323-1333.

53. Kurihara T, Brough DE, Kovesdi I, Kufe DW. Selectivity of a replication-competent adenovirus for human breast carcinoma cells expressing the MUC1 antigen. J Clin Invest. 2000;106: $763-771$.

54. Russell SJ. Gene therapy for cancer. Lancet. 1992;339:11091110.

55. Haag A, et al. Highly efficient transduction and expression of cytokine genes in human tumor cells by means of autonomous parvovirus vectors; generation of antitumor responses in recipient mice. Hum Gene Ther. 2000;11:597-609.

56. McCart JA, et al. Complex interactions between the replicating oncolytic effect and the enzyme/prodrug effect of vaccinia-mediated tumor regression. Gene Ther. 2000;7:12171223.

57. Dranoff G, Jaffee E, Lazenby A, et al. Vaccination with irradiated tumor cells engineered to secrete murine granulocytemacrophage colony-stimulating factor stimulates potent, specific, and long-lasting anti-tumor immunity. Proc Natl Acad Sci USA. 1993;90:3539-3543. 